\title{
Professora Vitória: um Chatbot para o ensino da Leitura
}

\author{
Daniel Brito Bulhões ${ }^{1}$, Luciana Pereira de Assis ${ }^{2}$, Adriana Nascimento Bodolay ${ }^{2}$, \\ Alessandro Vivas Andrade ${ }^{2}$, Cristiano Grijó Pitangui ${ }^{3}$ \\ ${ }^{1}$ Instituto Federal do Norte de Minas Gerais (IFNMG) - Campus Pirapora \\ Pirapora- - MG - Brasil \\ ${ }^{2}$ Universidade Federal dos Vales do Jequitinhonha e Mucuri (UFVJM) - Campus JK \\ Diamantina - MG - Brasil \\ ${ }^{3}$ Universidade Federal de São João Del-Rei (UFSJ) - Campus Alto Paraopeba \\ Ouro Branco - MG - Brasil \\ daniel.bulhoeseifnmg.edu.br, \\ \{lpassis, adriana.bodolay, alessandrovivas\}@ufvjm.edu.br, \\ pitangui.cristiano@ufsj.edu.br
}

\begin{abstract}
Chatbot is a digital conversation agent that has the ability to simulate a dialogue with a person in natural language. This capability makes it ideal for the application of Protocoled Reading, which involves asking questions about a text to assess the comprehension of what has been read and encouraging the reader to make inferences and predictions about what may follow. The purpose of this article is to show the implementation of a chatbot that applies the technique of reading protocol in a text without the presence of a teacher. Considering that chatbots are present in the most diverse areas, the research may contribute to the discussion of the use of chatbots applied to education, especially in the stimulus to reading, as a pedagogical support tool.
\end{abstract}

Resumo. Chatbot é um agente de conversação digital que tem a capacidade de simular um diálogo com uma pessoa em linguagem natural. Essa capacidade o torna ideal para a aplicação da Leitura Protocolada, que consiste na realização de perguntas sobre um texto para avaliar a compreensão do que foi lido e estimular o leitor a produzir inferências e fazer previsões sobre o que poderá vir em seguida. A proposta deste artigo é mostrar a implementação de um chatbot que aplique a técnica da Leitura Protocolada em um texto sem a presença de um professor. Considerando que os chatbots estão presentes nas mais diversas áreas, a pesquisa poderá contribuir na discussão do uso chatbots aplicados à educação, especialmente no estímulo à leitura, como ferramenta de apoio pedagógico.

\section{Introdução}

O constante avanço da tecnologia traz grandes propostas de apoio à educação e suas mais diversas modalidades. A implantação dos recursos computacionais à educação deve acontecer de forma cuidadosa, já que se trata de novas práticas e envolve quebras de paradigmas antigos.

O papel das TDICs (Tecnologias Digitais da Informação e Comunicação) é muito importante, pois trata de um conjunto de mídias e equipamentos digitais de apoio à 
educação, que, a cada dia vem sendo melhor explorados. Percebe-se que os estudantes atualmente estão inseridos em ambientes de maior interação, com várias fontes de informação, cooperação e colaboração. [Garcia et al. 2011] afirmam que "as tecnologias digitais interativas vislumbram a possibilidade de práticas não apenas mais dinâmicas, mas substancialmente modificadas". Nesse cenário, destacam-se o uso de agentes conversacionais digitais, também conhecidos como chatbots.

[Huang et al. 2007] definem como chatbots os agentes de conversação que interagem com os usuários sobre um determinado assunto ou tópico. Existem atualmente diversos chatbots, que empregam Inteligência Artificial e Processamento de Linguagem Natural, atuantes nas mais diferentes áreas, como em sistemas de telefonia, autoatendimento, entretenimento e e-commerce [Abbasi and Kazi 2014]. Na educação, eles tem sido utilizados nas mais variadas áreas de ensino e encontram-se diversas pesquisas e artigos que abordam o uso de robôs que simulam um diálogo humano no processo de ensino/aprendizagem, como a Prof ${ }^{a}$ Elektra [Leonhardt et al. 2003], o Blaze [Aguiar et al. 2011], o Tical [Moreno et al. 2015], o Metis [Lucchesi et al. 2018], o ChatterEDU [Martins and Martins 2017], dentre outros. A popularização de smartphones e computadores portáteis, aliados ao aumento de suas capacidades de processamento, oportunizaram a expansão e o desenvolvimento de novos chatbots [Moreno et al. 2015].

O ensino da leitura é complexo e constituído de muitos subprocessos na construção de uma ligação entre o leitor e o texto. O conhecimento desses subprocessos é essencial para que se aperfeiçoe o processo de ensino-aprendizagem da leitura [Coscarelli 1996]. Tornar o aluno um leitor proficiente é, sem dúvidas, um dos grandes desafios das escolas no brasileiras. O último exame do PISA detectou que, no Brasil, $50,1 \%$ dos estudantes estão abaixo de nível 2 de proficiência em leitura, e apenas $2 \%$ nos níveis 5 e 6, considerado recomendado em uma escala de 1 a 6 [OECD 2018]. Resultados assim demonstram que muitos alunos frequentam a escola e se formam sem se tornarem leitores autônomos e independentes, o que contribui para a formação de analfabetos funcionais.

Baseando-se nessa necessidade de melhora no ensino da leitura e, consequentemente, na formação de leitores proficientes, este trabalho propõe o desenvolvimento de um agente de conversação digital para auxiliar no ensino da leitura, denominado Professora Vitória. Entre as diversas técnicas de ensino-aprendizagem da leitura, escolhemos a Leitura Protocolada. Essa técnica consiste em uma atividade interativa que envolve o professor, o aluno e o texto, utilizada geralmente de forma presencial com a finalidade de desenvolver habilidades de predição e produção de inferências. O objetivo deste trabalho é avaliar se é possível a aplicação da Leitura Protocolada através de um chatbot. Para isso, foi desenvolvido um chatbot com uma base de conhecimento que seja capaz de interagir com o usuário sobre um texto piloto.

O artigo está organizado da seguinte maneira: na seção 2 é apresentada a técnica de leitura que será explorada neste trabalho e alguns conceitos importantes para o entendimento; na seção 3 é descrito o desenvolvimento e o funcionamento do chatbot; na seção seguinte, são apresentadas as avaliações e discutidos os principais resultados; por último, estão as considerações finais e trabalhos futuros. 
IX Congresso Brasileiro de Informática na Educação (CBIE 2020)

Anais do XXXI Simpósio Brasileiro de Informática na Educação (SBIE 2020)

\section{Formação de leitores e a Leitura Protocolada}

A formação de leitores definitivamente não é algo tão simples. A capacidade de se aprender a partir de um texto que leu faz o leitor ser autônomo ou não. Isso só é possível quando o leitor se questiona sobre sua própria compreensão, atualiza seu conhecimento, estabelece novas ligações entre o que foi lido e seu conhecimento prévio, aplicando em outros contextos o conhecimento que acabou de adquirir [Solé 1998].

Ler requer estratégias e essas são descritas por [Cantalice and Oliveira 2009] como métodos para abordagem de um problema e formas de atuar para alcançar um objetivo. Na leitura, as estratégias são planos flexíveis e adaptáveis usados pelos leitores para compreenderem diferentes textos. Cada um exige uma estratégia adequada para ser utilizada, contribuindo assim para facilitar a construção do conhecimento do texto lido. [Palinscar and Brown 1984] sugerem seis estratégias para o leitor obter um melhor aproveitamento em sua leitura:

1. Compreender os propósitos da leitura;

2. Ativar e aportar à leitura os conhecimentos prévios importantes para o conhecimento em questão;

3. Dirigir a atenção ao fundamental, em detrimento do que pode parecer mais trivial;

4. Avaliar a consistência interna do conteúdo expressado pelo texto e sua compatibilidade com o conhecimento prévio e com o "sentido comum";

5. Comprovar continuamente se a compreensão ocorre mediante a revisão e a recapitulação periódica e a auto interrogação;

6. Elaborar e provar inferências de diversos tipos, como interpretações, hipóteses e previsões e conclusões.

Dentre as estratégias propostas por [Palinscar and Brown 1984], destaca-se a produção de inferências, pois através delas o leitor poderá interpretar, prever, sugerir e concluir algo sobre um texto. [Coscarelli 1996] define que os processos inferenciais são essenciais para a leitura, podendo-se afirmar que quem não faz inferências não lê. A capacidade de realizar as inferências, deduzindo informações a partir de pistas fornecidas pelo ao texto, faz do indivíduo um leitor proficiente ou não. Uma forma de desenvolver a capacidade de fazer inferências é estimular a sua produção através de perguntas que exigem do leitor a realização de analogias e generalizações, em vez de perguntas em que as respostas estão explícitas no texto.

Baseado na estratégia de produção de inferências, existe uma técnica muito utilizada, chamada de Leitura Protocolada (ou pausa protocolada), que consiste em uma atividade que envolve a produção de inferências pelo leitor. O professor lê o texto dividindo-o em partes e, a cada parte lida, são feitas perguntas, para avaliar a compreensão do que foi lido e também para estimular os alunos a fazerem previsões sobre o que poderá vir no texto em seguida. Conforme se avança no texto, mais informações são consideradas, forçando o aluno a checar a compatibilidade de suas previsões com o que já se sabe do texto, trabalhando as relações de causa/consequência. A Leitura Protocolada pode ser aplicada a qualquer gênero textual, permitindo a produção de inferências e revelando ao leitor as suas estruturas preestabelecidas. Dessa forma, é possível que o leitor crie expectativas sobre quais tipos de informações teremos acesso em determinada parte do texto e melhore a sua capacidade de leitura [Coscarelli 1996]. 
A utilização da técnica Leitura Protocolada tem se mostrado eficiente no estímulo à produção de inferências [Dell'isola 1988, Coscarelli 1996, Fernandes 2015, dos Santos and da Rosa Marozo 2016], conseguindo chamar a atenção dos leitores não apenas para a importância de informações explícitas no texto, mas também das implícitas, utilizando para isso informações extratextuais, como o seu conhecimento de mundo. Apesar de não ser uma técnica recente, sua aplicação ainda é feita geralmente de modo presencial em sala de aula. Seguindo as tendências de educação à distância e uso de recursos digitais na educação, a ferramenta questionário do Moodle já foi utilizada para a aplicação da técnica [Bodolay et al. 2011]. Entretanto, a utilização de chatbot para a aplicação da Leitura Protocolada ainda não foi explorada, o que traz ineditismo para este trabalho.

\section{Construção da Atividade Leitura Protocolada}

A elaboração da atividade Leitura Protocolada pode ser dividida em três etapas: escolha do texto, divisão do texto e elaboração das perguntas. A escolha do texto é muito importante para o êxito dessa atividade, pois apesar de poder ser aplicada a qualquer gênero textual, a Leitura Protocolada é melhor explorada em textos surpreendentes, piadas e textos da literatura fantástica [Coscarelli 1996]. Nesse contexto, o texto escolhido para ser explorado nessa atividade foi "A armadilha" do autor de literatura fantástica Murilo Rubião, cuja as obras são caracterizadas por serem surpreendentes e surreais.

A divisão do texto deve acontecer em pontos que criam expectativas no leitor e de forma que a disposição das perguntas não fique concentradas em apenas um trecho. No nosso exemplo, o texto foi dividido em nove partes menores e para cada uma delas foram atribuídas perguntas preditivas e inferenciais. Ao todo, são 36 perguntas que além de provocar a curiosidade do leitor, possuem a função de instigar o leitor a estabelecer ligações a fatos reais, contextos, emoções dos personagens e construir hipóteses. A Figura 1 seguir apresenta um trecho do texto e as peguntas construídas para esse trecho.

"Parou diante do último escritório e perdeu algum tempo lendo uma frase, escrita a lápis, na
parede. Em seguida passou a mala para a mão esquerda e com a direita experimentou a
maçaneta, que custou a girar, como se há muito não fosse utilizada. Mesmo assim não
conseguiu franquear a porta, cujo madeiramento empenara. Teve que usar o ombro para
forçá-la. E o fez com tamanha violência que ela veio abaixo ruidosamente. Não se
impressionou. Estava muito seguro de si para dar importância ao barulho que antecedera a
sua entrada numa saleta escura, recendendo a mofo. Percorreu com os olhos os móveis, as
paredes. Contrariado, deixou escapar uma praga. Quis voltar ao corredor, a fim de recomeçar
a busca, quando deu com um biombo. Afastou-o para o lado e encontrou uma porta
semicerrada. Empurrou-a. la colocar a mala no chão, mas um terror súbito imobilizou-o:
sentado diante de uma mesa empoeirada, um homem de cabelos grisalhos, semblante
sereno, apontava-lhe um revólver. Conservando a arma na direção do intruso, ordenou-lhe
que não se afastasse."
Perguntas:
- Que frase Alexandre teria lido na parede?
- Quem poderia ter escrito?
Que relação você estabelece agora entre o título e o que ocorre nessa passagem do texto?
- Quem poderia ser esse "homem de cabelos grisalhos"?
- Você acha estaria com um revólver?
- O que você espera que aconteça no desenrolar do texto?

Figura 1. Trecho do texto "A Armadilha"[Rubião 1998] e perguntas da Leitura Protocolada.

A intenção da maioria das perguntas que são realizadas na atividade não é obter 
respostas exatas ou consideradas corretas, pois não tratamos aqui de um questionário avaliativo convencional. O objetivo da Leitura Protocolada é incentivar o leitor a produzir inferências, permitindo que possa arriscar, imaginar e errar se for preciso, para construir ou reconstruir suas próprias hipóteses de leitura. Portanto, perguntas que se referem ao tempo verbal ou gêneros textuais possuem respostas esperadas, enquanto perguntas que se referem a opinião do leitor ou às suas expectativas não podem possuir respostas exatas, uma vez que podem variar de pessoa para pessoa.

\section{Criação do Chatbot Professora Vitória}

O chatbot desenvolvido neste trabalho foi nomeado "Professora Vitória" e é composto por três elementos principais. O primeiro é a interface, responsável por promover a interação entre o programa e o usuário. Neste trabalho foi utilizada uma interface bastante simples, desenvolvida na linguagem JAVA e organizada da seguinte forma: à esquerda está destinado o espaço para exibição do trecho do texto a ser lido pelo usuário; à direita, está localizado um balão onde é exibido a pergunta do robô e outro onde é exibida a última resposta do usuário; abaixo dos balões de falas estão a caixa de diálogo para que o usuário digite a sua resposta e um botão de enviar. A Figura 2 ilustra a interface da Professora Vitória:

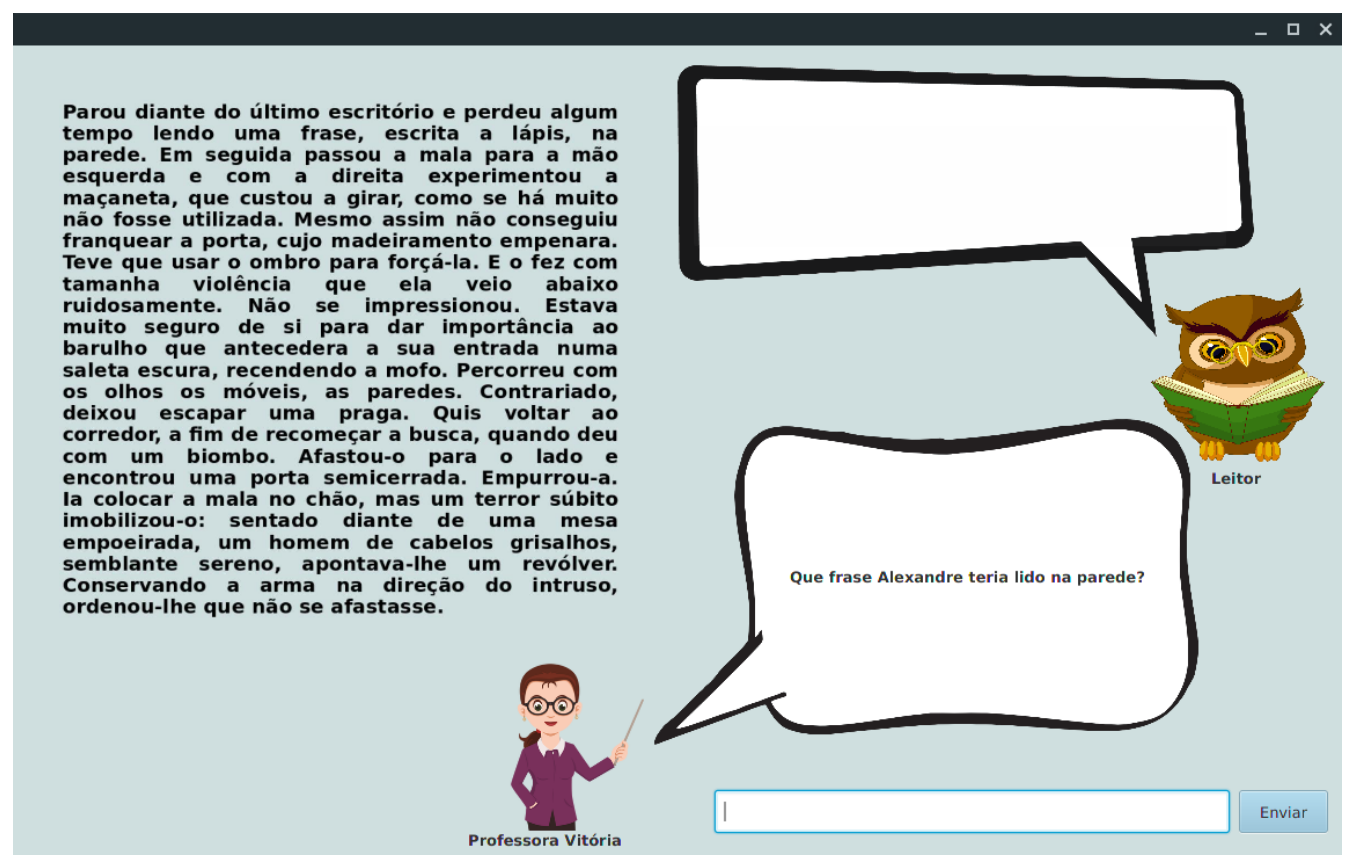

Figura 2. Interface do chatbot Professora Vitória.

O segundo elemento é a base de conhecimento, onde são cadastradas todas as possíveis respostas que o chatbot irá retornar. No caso deste projeto, para a construção da base de conhecimento foi utilizada AIML (Artificial Intelligence Markup Language), que é uma linguagem de marcação própria para isso. [Shawar and Atwell 2007] explicam que a estrutura da linguagem AIML é formada por tags e consiste em objetos de dados, compostos de unidades chamadas tópicos e categorias. O tópico é opcional e relaciona um conjunto de categorias em um mesmo lugar. A categoria é uma regra, que define uma saída para cada tipo de entrada de texto. 
As principais tags utilizadas neste trabalho são: Category: é a unidade de conhecimento básica do AIML, composta de uma possível mensagem de entrada e uma resposta de saída; Pattern: é um possível padrão de mensagem digitado pelo usuário; Template: é a resposta que será dada pelo chatbot ao usuário; That: determina para qual pergunta uma determinada sentença digitada pelo usuário se refere. A Figura 3 mostra um trecho do código da Professora Vitória em AIML:

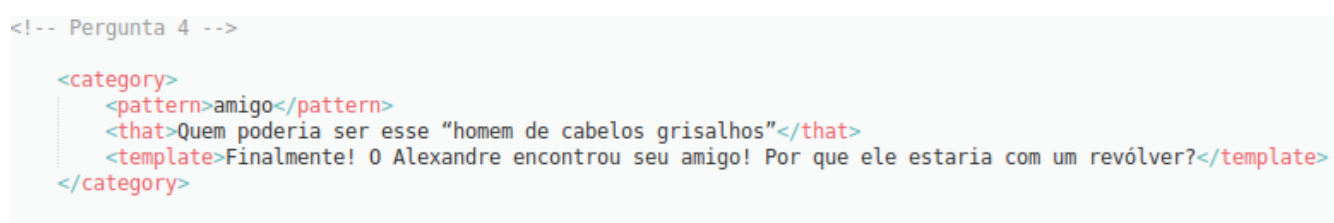

Figura 3. Parte do código AIML do chatbot Professora Vitória.

O terceiro elemento é o interpretador, que tem a função de analisar o texto escrito em linguagem natural pelo usuário e buscar na base AIML a melhor resposta. Existem diversos como: Program Z, presente no site www.pandorabots.com; Program AB do Google; Program $M$ para a linguagem SETL; "PHiliP" ou Program E para implementação em PHP, dentre outros. Neste trabalho, foi utilizado o Program $A B$ por permitir a integração com JAVA e ter obtido os melhores resultados em testes durante o processo de desenvolvimento do presente trabalho. A Figura 4 mostra a organização deste chatbot em forma de fluxograma:

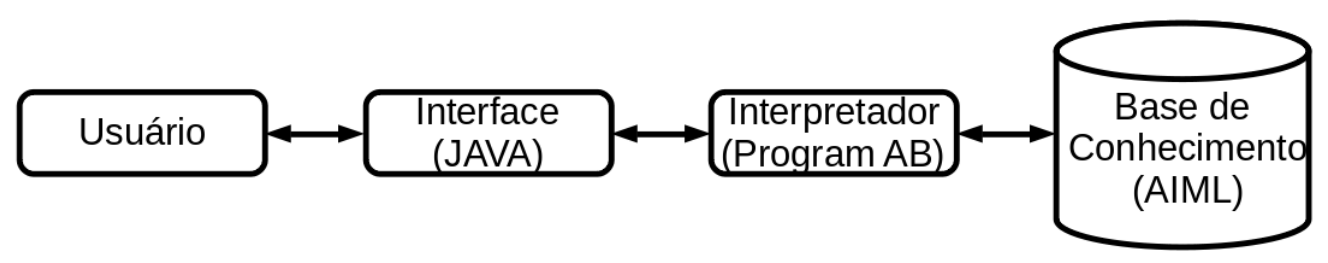

Figura 4. Organização do chatbot Professora Vitória.

É importante ressaltar que este chatbot trabalha em uma perspectiva um pouco diferente de outros, pois é ele que apresenta a pergunta ao usuário buscando sempre propor o diálogo e não apenas responde perguntas. Por possuir tal característica, a implementação da base de conhecimento torna-se um processo mais demorado, uma vez que o número de respostas possíveis que podem ser digitadas pelo usuário pode ser imensurável. Considerando essa questão, a base de conhecimento foi construída a partir das respostas dadas por alunos em atividades presenciais já realizadas anteriormente com esse mesmo texto, selecionando as que mais se repetem para cada pergunta.

O ideal é que o agente de conversação sempre consiga retornar uma resposta compatível com o que o usuário digitou e por isso a base de conhecimento deve ser muito bem trabalhada. Entretanto, caso uma determinada sentença não tenha uma resposta compatível, o chatbot retornará uma resposta genérica e fará a próxima pergunta para dar continuidade ao diálogo.

\section{Avaliação e Resultados}

O chatbot Professora Vitória foi disponibilizado para a utilização por profissionais da educação (professores e pedagogos) e alunos de graduação para que fosse possível captar 
algumas informações que validassem o projeto. A partir do recurso de registro de $\log s$ presente neste programa, foi possível obter as respostas dos usuários das perguntas da atividade de Leitura Protocolada. Os arquivos continham registros de 50 usuários, que foram utilizados para análise neste trabalho. Ao final da atividade, o chabot requisitava um feedback do usuário sobre a utilização do software. Foram feitas as seguintes perguntas:

1. O que você achou da Professora Vitória?

2. Após a interação com o chatbot, você acha que a houve alguma contribuição para a compreensão do texto?

3. Quais possíveis melhorias no programa você sugere?

A receptividade dos entrevistados em relação ao programa foi muito boa e todos mostraram-se muito satisfeitos em utilizá-lo, relatando que foram instigados a usar a imaginação, despertar a curiosidade e incentivados a dar continuidade a história. Dentre os 50 leitores envolvidos nesta pesquisa, 48 afirmaram que perguntas feitas pela Professora Vitória contribuíram de forma relevante para o entendimento do texto. Vale ressaltar a recorrência dos comentários a respeito da capacidade do programa em envolver o leitor no enredo da história, despertar sua curiosidade, instigar o uso da imaginação e contribuir para o levantamento de hipóteses. Tais reações são esperadas em uma leitura protocolada, o que demonstra o grande potencial desta ferramenta eletrônica, mesmo que esteja ainda em fase de aperfeiçoamento.

Os participantes da pesquisa também identificaram pontos negativos nesse chatbot e 39 participantes sugeriram melhorias. Entre as sugestões mais recorrentes e relevantes, destacamos quatro: a primeira, apontada por nove entrevistados, é a melhoria no vocabulário, principalmente no que se refere aos sinônimos, pois o programa às vezes não interpreta um termo digitado como deveria, rejeitando palavras similares em algumas sentenças; a segunda sugestão refere-se à clareza e objetividade das perguntas que, para 12 entrevistados, precisa melhorar; a terceira, citada por seis participantes, é sobre a possibilidade de inserção de recursos audiovisuais, como ilustrações dos trechos do texto e áudio nas perguntas do chatbot; por último, quatro participantes sugeriram que a atividade ou o texto fossem mais curtos. Os resultados estão condensados na Tabela 1:

Tabela 1. Tabela 1 - Sugestões para o Chatbot Professora Vitória

\begin{tabular}{l|c}
\multicolumn{1}{c|}{ Sugestão } & Número de ocorrências \\
\hline Melhoria no vocabulário/repertório de sinônimos & 9 \\
\hline Realizar perguntas mais diretas & 7 \\
\hline Inserção e imagens ou recursos de áudio & 6 \\
\hline Realizar perguntas mais explicativas & 5 \\
\hline Atividade ou texto mais curtos & 4 \\
\hline Outras & 8 \\
\hline Sem sugestões & 11 \\
\hline
\end{tabular}

A avaliação geral sobre a Professora Vitória foi muito positiva, o que sinaliza um grande potencial da ferramenta na aplicação da leitura protocolada. O chatbot conseguiu realizar as perguntas sobre o texto e captar boa parte das respostas do usuário, dando continuidade no diálogo de forma satisfatória. Entretanto, nesses primeiros testes realizados, foi possível perceber a necessidade de incrementação da base de conhecimento para aperfeiçoamento deste chatbot. 
Analisando os $\log s$ das respostas da Leitura Protocolada, percebe-se que a grande variação de respostas, tanto nas perguntas de natureza preditiva, referentes a o que o leitor espera que aconteça, quanto nas perguntas de natureza inferencial, caracterizadas pela busca do não dito a partir do dito [Oliveira 2010]. A pergunta preditiva "O que você pode esperar de um texto cujo o título é A armadilha?", por exemplo, obteve nesta pesquisa sete variações de respostas: um conto de terror, história de aventura, uma cilada, uma pegadinha, armações, uma parábola e história de suspense. Na pergunta inferencial referente ao trecho da Figura 1 "Por que ele estaria com o revólver?", houve 10 variações de respostas. Entende-se que esse número variados de respostas está ligado diretamente ao perfil de cada entrevistado, grau de escolaridade, contexto socioeconômico, experiência de vida, a qual pergunta e em que momento do texto a pergunta foi colocada, pois todos esses fatores podem influenciar a percepção do leitor.

Se a ferramenta for utilizada por diversas pessoas, é possível analisar as respostas que mais se repetem, as que não estão previstas na base de conhecimento e onde o leitor pode perder o interesse na utilização do programa, tendo como base as perguntas que não foram respondidas ou foram de forma displicente. Esse é um processo parecido com o que já acontece na aplicação da leitura protocolada de forma presencial em sala de aula, pois, ao longo do tempo, o professor percebe quais perguntas são relevantes ou não para a atividade e aperfeiçoa a técnica. No caso do chatbot, a atividade será melhorada com base na análise do arquivo de backup, que contém todas as respostas que foram dadas pelos usuários. A tendência é que a Professora Vitória fique mais eficiente a cada versão, pois terá sua base de conhecimento melhorada e, consequentemente, sua capacidade de diálogo.

Da perspectiva do programador, essa análise é muito importante para a construção/atualização do programa. Para o professor, as respostas digitadas pelos leitores possibilitam que ele verifique os erros ortográficos e vocabulário de cada estudante. Nesse experimento, por exemplo, 34 dos 50 participantes escreveram suas respostas com pelo menos um erro ortográfico em algum momento. Essas informações são importantes para que o professor possa adaptar suas aulas, mudar a estratégia de ensino-aprendizagem, trabalhando de forma mais efetiva nas fragilidades de uma determinada turma ou de um aluno específico.

O contexto de uma sala de aula, os improvisos diante de algum fator externo ou a um questionamento de um aluno trazem características únicas para uma aula presencial e, consequentemente, dificulta a programação de um agente digital como a Professora Vitória para a aplicação de uma atividade tão interativa. Mesmo assim, o uso de um chatbot na leitura protocolada demonstrou uma vantagem significativa para a sua aplicação: a participação individual de todos os alunos. Em um contexto presencial e coletivo, como em uma sala de aula, alguns estudantes participam mais, outros menos e outros nem participam, seja por medo de errar, vergonha ou falta oportunidade. A ferramenta digital, por sua vez, oferece ao aluno um atendimento individual, além de ficar disponível para utilização a qualquer momento do dia e em qualquer lugar com um computador.

\section{Considerações finais e trabalhos futuros}

Este trabalho experimental mostra que é possível a aplicação da Leitura Protocolada por meio de um chatbot. As primeiras impressões sobre a utilização desse agente conversaci- 
onal digital vem contribuir nas discussões sobre a importância do ensino das estratégias e técnicas de leitura, bem como no uso didático de ferramentas digitais.

Entretanto, existem alguns desafios que devem ser superados. Um deles é a construção da base de conhecimento, que é complexa, única e não pode ser aproveitada em outros textos. Com a retroalimentação da base de conhecimento, é possível fazer ajustes constantes de forma a acrescentar e abranger um maior número de respostas, aprimorando o diálogo com o leitor. Por se tratar de um programa de código aberto e gratuito, é possível que outros programadores possam realizar melhorias e aplicá-las a outras obras literárias. Outro desafio é facilitar para um professor que não seja programador, consiga criar seu próprio chatbot, com textos diferentes e suas próprias perguntas.

É importante ressaltar que o papel do professor é, e sempre será, importante para a formação dos discentes e não há intenção nenhuma de excluir sua figura do processo de ensino-aprendizagem. A Leitura Protocolada é uma metodologia para o ensino de leitura, mediada sempre por um professor de maneira presencial. Porém, em ambientes em que não temos um professor presencial, como em ambientes virtuais de aprendizagem ou no Ensino à Distância, é necessário que existam outras formas para que o professor consiga trabalhar com essa metodologia.

O chatbot Professora Vitória apresenta-se como um recurso digital disponível, podendo ser explorado tanto no ensino presencial, quanto em EAD. Essa disponibilidade é um fator que abre novas possibilidades para o professor conduzir atividades com seus alunos utilizando essa ferramenta digital como mais um recurso didático. A adição de novos recursos, como a disponibilização on-line, aplicativo de celular e inserção de imagens ilustrativas nos trechos do texto são parte da continuidade deste projeto, bem como a utilização de técnicas de Processamento de Linguagem Natural para aprimorar o diálogo e tratar novas sentenças que não estão embutidas na base do conhecimento.

Vislumbramos a possibilidade em um futuro próximo de que textos e livros digitais possam já vir inclusos com um chatbot para a aplicação da leitura protocolada. Diversas obras disponíveis com esse recurso, principalmente nos primeiros anos de escolarização, proporcionaria um acervo maior aos educadores e consequentemente uma melhor abordagem nos processos de ensino-aprendizagem da leitura e suas estratégias.

\section{Agradecimento}

Agradecemos ao Programa de Bolsas para Qualificação de Servidores (PBQS-IFNMG), do qual o primeiro autor tem sido bolsista.

\section{Referências}

Abbasi, S. and Kazi, H. (2014). Measuring effectiveness of learning chatbot systems on student's learning outcome and memory retention. Asian Journal of Applied Science and Engineering, 3(2):251-260.

Aguiar, E. V. B., Tarouco, L. M. R., and Reategui, E. B. (2011). A construção do conhecimento matemático com engajamento e aprimoramento de habilidades cognitivas apoiada por um agente conversacional. RELATEC.

Bodolay, A. N., de Oliveira, E. F., Martins, M. E., and Cunha, Y. K. (2011). Ensino de estratégias de leitura mediadas por computador: experiência do projeto praler. Signo, 36(61):356-369. 
IX Congresso Brasileiro de Informática na Educação (CBIE 2020)

Anais do XXXI Simpósio Brasileiro de Informática na Educação (SBIE 2020)

Cantalice, L. M. d. and Oliveira, K. L. d. (2009). Estratégias de leitura e compreensão textual em universitários. Psicologia escolar e Educacional, 13(2):227-234.

Coscarelli, C. V. (1996). O ensino da leitura: uma perspectiva psicolingüística. Boletim da Associação Brasileira de Linguística, pages 163-174.

Dell'isola, R. L. P. (1988). Leitura: inferências e contexto sócio-cultural.

dos Santos, J. and da Rosa Marozo, L. F. (2016). Contribuições para o desenvolvimento do leitor crítico: desmistificando o preconceito. CCNExt-Revista de Extensão, 3:341345 .

Fernandes, V. L. R. (2015). Pausa protocolada na leitura: ensinando a fazer inferências.

Garcia, M. F., Rabelo, D. F., da Silva, D., and do Amaral, S. F. (2011). Novas competências docentes frente às tecnologias digitais interativas. Teoria e Prática da Educação, 14(1):79-87.

Huang, J., Zhou, M., and Yang, D. (2007). Extracting chatbot knowledge from online discussion forums. In IJCAI, volume 7, pages 423-428.

Leonhardt, M. D., Castro, D. D. d., Dutra, R. L. d. S., and Tarouco, L. M. R. (2003). Elektra: Um chatterbot para uso em ambiente educacional. RENOTE: revista novas tecnologias na educação [recurso eletrônico]. Porto Alegre, $R S$.

Lucchesi, I. L., da Silva, A. R., Abreu, C., and Tarouco, L. M. R. (2018). Avaliação de um chatbot no contexto educacional: Um relato de experiência com metis. RENOTE, $16(1)$.

Martins, J. and Martins, C. (2017). Geração de perguntas e respostas para a base de conhecimento de um chatterbot educacional (application to generate questions and answers for an educational chatterbot)[in portuguese]. In Proceedings of the 11th Brazilian Symposium in Information and Human Language Technology, pages 22-26.

Moreno, F., Manfio, E., Barbosa, C. R., and Brancher, J. D. (2015). Tical: Chatbot sobre o atlas linguístico do brasil no whatsapp. In Brazilian Symposium on Computers in Education (Simpósio Brasileiro de Informática na Educação-SBIE), volume 26, page 279.

OECD (2018). Pisa 2018: Technical report. Disponível em: http://www.oecd.org/pisa/PISA\%202018\%20Insights\%20and\%20Interpretations\%20 FINAL\%20PDF.pdf. Acesso em: 13 de julho de 2020.

Oliveira, L. A. (2010). O ensino pragmático da literatura. __-_-_. Coisas que todo professor de português precisa saber: a teoria na prática. São Paulo: Parábola Editorial, pages 171-194.

Palinscar, A. S. and Brown, A. L. (1984). Reciprocal teaching of comprehension-fostering and comprehension-monitoring activities. Cognition and instruction, 1(2):117-175.

Rubião, M. (1998). Contos reunidos. Editora Atica.

Shawar, B. A. and Atwell, E. (2007). Chatbots: are they really useful? In Ldv forum, volume 22, pages 29-49.

Solé, I. (1998). Estratégias de leitura. trad. Cláudia Schilling, 6. 